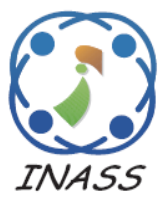

\title{
DC Motor Parameters Identification Using Farmland Fertility Optimizer
}

\author{
Hady Abdel-Maksoud ${ }^{1,2 *}$ \\ ${ }^{1}$ Faculty of Engineering, Menoufia University, Egypt \\ ${ }^{2}$ Faculty of Engineering, Northern Border University, Saudi Arabia \\ * Corresponding author's Email: hady_elgendy@yahoo.com
}

\begin{abstract}
DC motor drive is still widely employed in manufacturing and transportation systems. The control schemes require an accurate model for recognizing the motor performance. Some or completely the motor parameters may be unknown for the designer. In this research, the application of farmland fertility optimization (FFO) algorithm to identify the parameters of DC motor is presented. This paper purposes to develop an efficient DC model to characterize its performance under various working conditions. The experimental system to validate the proposed method in identification the motor parameters has been built. The parameters of DC motor have been estimated based the actual motor speed response. Then, the proposed technique has been examined at different motor operation as starting, load change and speed change. Subsequently, the experimental results are compared with results obtained from the FFO. It is found that a good convergence between the actual and estimated results is appeared and the convergence rate is continuously decreased until highly acceptable value. Furthermore, a comparison between the results of FFO and those based recent optimization technique has been occurred. It can be assured that the FFO performs well and has good capability of recognizing the unknown DC motor parameters with fewer errors.
\end{abstract}

Keywords: DC Motor, Parameter identifications, Farmland fertility optimizer.

\section{Introduction}

Although the thermal and the mechanical restrictions, the direct current (DC) motors remain have been widely used in different applications such as electrical vehicles, water pump, medical equipments, robots, mechatronic and mining machines [1-3]. For implementing a DC motor in sophisticated control systems, both electrical and mechanical parameters must be identified precisely $[4,5]$. So, there is a great attention in the procedure for determining the parameters of DC motor. Furthermore, the motor specifications which are given by manufacturer may be considered inadequate, especially for inexpensive DC motors that resort to tolerate comparatively big toleration in their mechanical and electrical parameters [6, 7].

Various methods and optimization techniques have been developed for solving the problem of parameters identification in the DC motor [8-10]. In [11] the authors proposed the methodology of Flower
Pollination Algorithm (FPA) to estimate the parameters of DC motor, the algorithm has a good idea, but the results have a high error in convergent rate and the technique is just applied at starting condition. The Nelder-Mead simplex method is utilized in [12] to define the required parameters. However, a few results are presented beside it is shortened to present the coveted outcomes. Adaptive Tabu Search (ATS) technique is applied in [13] for identification of the parameters of separately excited DC motor. The technique is unpretentious, but the inaccuracy is appeared because it was limited in studying the problem. So, it has not assured to converge, nor essentially generate the minimum residue error. W. Wu employed speed step responses [14]; this is a tedious process where the machine response must be analyzed with and without torque disturbances. Virtual instrumentation was proposed in [15] to predict the motor parameters. Unfortunately, the implementation of this method based simulation structure. Hence, the accurate estimation of parameters is not ensured. In [16] wavelet functions 
are applied for predication the parameters of DC motor with independent excitation. The accuracy is perspicuous, but it has been examined solely at starting. M. Lankarany and A. Rezazade are utilized Genetic Algorithm in [17]. In spite of it has good performance, it requires huge computational load because of the fundamental property of populationbased investigation

Lately, Farmland fertility optimizer [FFO] methodology is employed for solving the continuous optimization problems in the electrical system components [18]. It has advantages of less complexity, applicable and powerful algorithm when compared with conventional optimization methods [19]. FFO performs well in identification the parameters of unknown system such as photovoltaic array [20]. Hence, this paper is committed to investigate the FFO algorithm for identification of DC motor parameters.

The paper's objectives can be presented as follow:

1) Proposing FFO for optimally generate the unknown parameters of DC motor.

2) Implementation of the experimental test bed.

3) Verification of the proposed method by comparing its results with experimental one.

4) Comparison between FFO and recent optimizers based - results in literature.

The paper is arranged as follows: Section 1 exhibits a brief introduction and literature review. The dynamic model of DC motor with independent excitation is presented in Section 2. FFO is described in Section 3. The algorithm of parameters identification is explained in Section 4. Section 5 introduces the experimental system. Experimental results and discussion are displayed in Section 6. Conclusion and references are appeared in Sections 7 and 8 respectively.

\section{Modelling of DC motor}

In this part of the paper, the dynamic model of DC motor with separate excitation is introduced as follow:

$$
\begin{gathered}
V=R_{a} i_{a}+L_{a} \frac{d i_{a}}{d t}+E_{b} \\
V_{f}=R_{f} i_{f}+L_{f} \frac{d i_{f}}{d t} \\
E_{b}=K_{V} \cdot \omega_{m}
\end{gathered}
$$

Where,

$V:$ armature voltage [V]

$V_{f}$. excitation voltage $[\mathrm{V}]$
$E_{b}$ : back emf $[\mathrm{V}]$

$i_{a}:$ armature current $[\mathrm{A}]$

$i_{f}$ : excitation current $[\mathrm{A}]$

$R_{a}$ : armature resistance [ $\left.\Omega\right]$

$R_{f}$ : field resistance $[\Omega]$

$L_{a}$ : armature inductance $[\mathrm{H}]$

$L_{f}$ : field inductance $[\mathrm{H}]$

$K_{v}$ : motor constant coefficient [V.s/rad]

$\omega_{m}:$ motor angular speed $[\mathrm{rad} / \mathrm{s}]$

The torque equilibrium equation for DC motor can be written as:

$$
T_{d}=J \frac{d \omega_{m}}{d t}+B \omega_{m}+T_{L}
$$

Where,

$T_{d}$ : developed torque [N.m]

$T_{L}$ : load torque [N.m]

$J$ : moment of inertia coefficient [Kg.m2]

$B$ : viscous friction coefficient [N.m.s/rad]

The speed $\omega_{m}$ and armature current $i_{a}$ are taken as the state variables while the supply voltage $V_{s}$ and load torque $T_{L}$ are chosen as state inputs for derivation the state-space model for the dc motor with independent excitation as indicated in Eq. (5).

$$
\left[\begin{array}{c}
\frac{d i_{a}}{d t} \\
\frac{d \omega_{m}}{d t}
\end{array}\right]=\left[\begin{array}{cc}
-\frac{R_{a}}{L_{a}} & -\frac{K_{V}}{L_{a}} \\
\frac{K_{V}}{J} & -\frac{B}{J}
\end{array}\right]\left[\begin{array}{c}
i_{a} \\
\omega_{m}
\end{array}\right]+\left[\begin{array}{c}
\frac{1}{L_{a}} \\
-\frac{1}{J}
\end{array}\right]\left[\begin{array}{l}
V_{s} \\
T_{L}
\end{array}\right]
$$

It can be recognized the graphical interpretation of state space equation in the block diagram in Fig. 1. Based on block diagram of the DC motor, it can be determined the transfer function in the hypothesis of neglecting the disturbance torque as indicated in the following equation:

$$
\frac{\omega_{m}(s)}{V_{S}(s)}=\frac{K_{V}}{\left(R_{a}+L_{a} s\right)(B+J s)+K_{V}^{2}}
$$

Referring to Eq. (6), it is seen that there are number of parameters namely $R_{a}, L_{a}, B, J$ and $K_{V}$ are required to be defined. FFO is applied for adjusting the coefficients to keep finest values inside their bottom and top bounds. Optimization is performed to ensure an exact modelling of DC motor.

\section{Farmland fertility optimizer}

The idea of farmland fertility optimizer (FFO) is come from the way with which farmers define the soil goodness (SG) of each section of farmland.

Composition of the soil and adding of certain materials are the basic reasons for the differences in SG. Hence, farmers use special materials to raise SG level in their farmland. In fact, adding these materials 


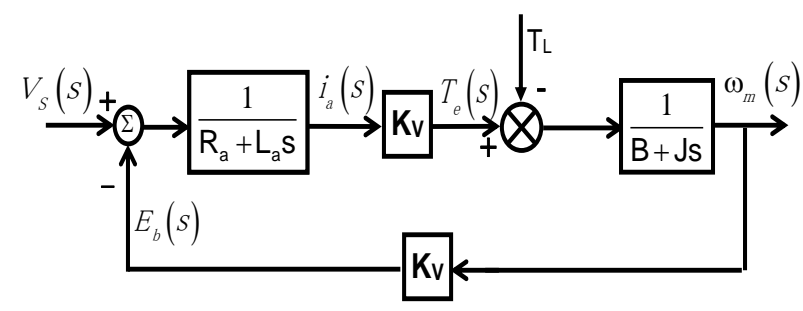

Figure. 1 The block diagram of the DC motor with independent excitation

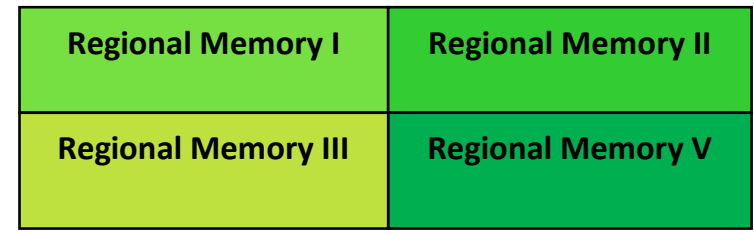

Figure. 2 Example for divided farmland, local memory, and global memory

to the soil may improve or lower the SG level. The definition of the materials needed to upgrade SG is based on past experiences, which are kept in local and global memories, depending on farmers' expertise with soil natures and past outcomes of SG upgrade. If the farmland is supposed to be divided to four sections as shown in Fig. 2, each section has its distinct local memory and a global memory and section III has the lowest SG.

\subsection{Objective function and restrictions}

The objective function (OF) relies on reduction of the root of the mean squared errors (RMSE) between the actual and the calculated speeds for identification of the DC motor parameters, as indicated in (7).

$$
O F=\min \left(\sqrt{\frac{1}{N} \sum_{k=1}^{N}\left(\omega_{a c t}(k)-\omega_{c a l}(k)\right)^{2}}\right)
$$

Where $\mathrm{N}$ denotes the quantity of measurement points, $\omega_{\text {act }}$ and $\omega_{\text {cal }}$ symbolize the actual and calculated motor speed, respectively. The OF is presented for the constraints that are defined by minimum and highest values of $\left(R_{a}, L_{a}, B, J, K_{V}\right)$

\subsection{Procedure of farmland fertility optimizer}

The procedure steps of FFO are illustrated in Fig. 3. Initially, the FFO parameters have been identified. Based on the number of farmland sections (n) and the quantity of available solutions in each section (m), the total quantity of population $\mathrm{N}_{\text {pop }}$ in the seeking range has been determined in the first step.

$$
N_{p o p}=m \cdot n
$$

To randomly generate each of the solutions within the scope of research, the following equation is used

$$
Y_{i j}=M_{i}+\operatorname{rand} .\left(U_{i}-M_{i}\right)
$$

Where $i=\{1,2, \ldots, m\}, j=\{1,2, \ldots, n\}, \mathrm{M}_{\mathrm{i}}$ and $\mathrm{U}_{\mathrm{i}}$ are minimum and maximum values of solution $\mathrm{Y}$, consecutively, rand is a random value between 0 and 1. Also, the solutions obtainable within the scope of the research are fully computed in this step according to $\mathrm{OF}$ and the type of optimization problem taken into account the upper and the lower limits of the solutions.

Secondly, definition for each section of farmland solutions is determined as follows:

$$
\begin{aligned}
& \text { Section }_{s}=y\left(b_{i}\right), b=n .(S-1)+1: n . S \\
& \quad \text { Where } S=\{1,2,3, \ldots m\}, i=\{1,2,3, \ldots m\} .
\end{aligned}
$$

The SG for each section is determined depending on the average of the current solutions in each section.

In the third step, the finest solutions for each section are kept in its local memory but the finest solutions of overall sections are kept in the global memory. The amount of solutions in the local memory $\left(\mathrm{Q}_{\mathrm{loc}}\right)$ and the amount of solutions in the global memory $\left(\mathrm{Q}_{\mathrm{glob}}\right)$ are presented in (11) and (12), respectively:

$$
\begin{gathered}
Q_{l o c}=\operatorname{round}(x . n), 0<x<0.1 \\
Q_{\text {glob }}=\operatorname{round}\left(x . N_{\text {pop }}\right), 0<x<0.1
\end{gathered}
$$

Based on the fitness and the suitability, the solutions are placed into these memories. After updating these memories, the best and lowest sections have been recognized.

In the final step, the section which has the lowest SG will have the major differences and its solutions are combined with an obtainable solution in global memory as illustrated in the following equations

$$
\begin{gathered}
Y_{\text {new }}=a \cdot\left(Y_{i j}-Y_{Q_{g l o b}}\right)+Y_{i j} \\
a=\alpha \cdot \operatorname{rand}(-1,1)
\end{gathered}
$$

Where, $Y_{\text {new }}$ is the new solution, $Y_{i j}$ denotes the solution in lowest section of farmland which is selected to perform variations, $Y_{\text {glob }}$ is randomly chosen from the current solutions within the global memory and $\alpha$ is a parameter of FFO which is firstly adjusted between 0 and 1 . 
After implementing the differences in the lowest section of farmland, the remaining portions need to be integrated with solutions obtainable within the full scope of the research. The solutions can be obtained in the other sections by (15) and (16).

$$
\begin{gathered}
Y_{\text {new }}=a \cdot\left(Y_{i j}-Y_{u j}\right)+Y_{i j} \\
a=\beta \cdot \operatorname{rand}(0,1)
\end{gathered}
$$

Where $Y_{u j}$ is randomly chosen from the current solutions within the entire search extent and $\beta$ is a parameter of FFO which is primarily adapted between 0 and 1 .

Then, the solutions within the scope of the research are calculated entirely according to the objective function. Regardless of the amount of parts, this step is performed on all solutions obtainable within the scope of the research. The value of the fitness and the appropriateness of each of the current solutions are determined in the scope of the research. FFO completed or maintained to maximum number of iterations $\left(\mathrm{N}_{\max }\right)$ as per final conditions met. Fig. 3 shows a flowchart of FFO.

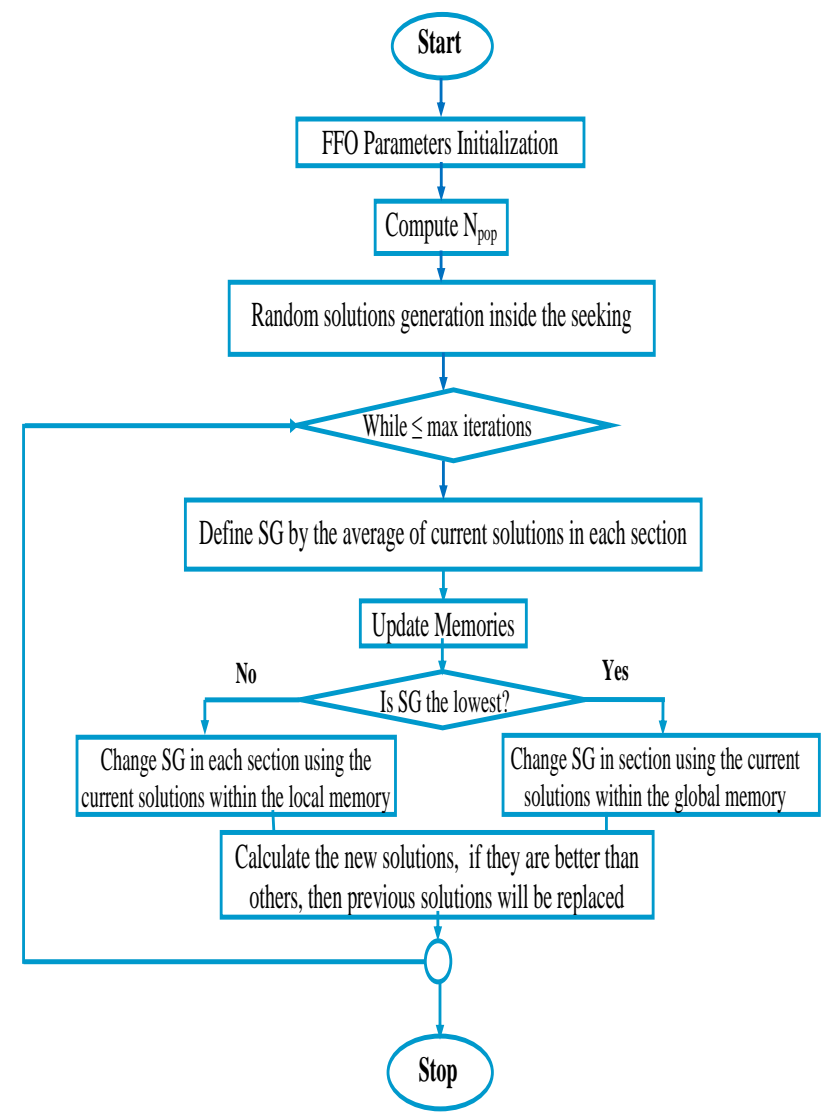

Figure. 3 Flowchart of FFO

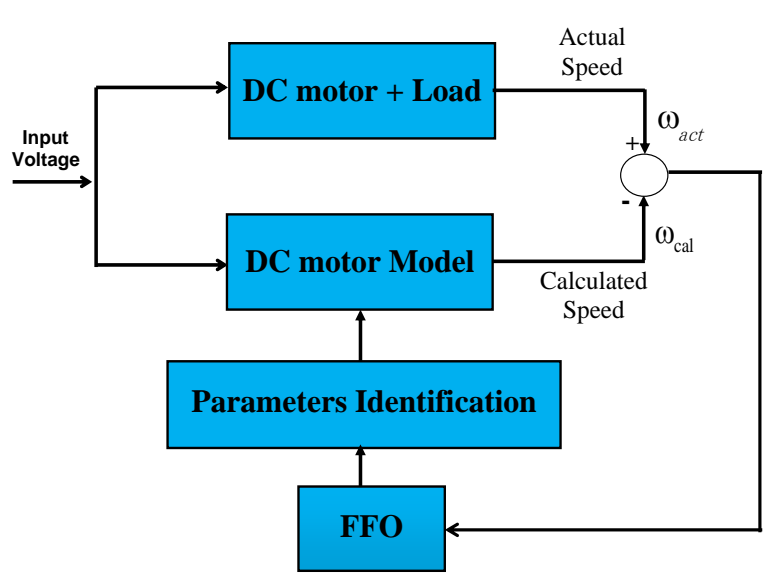

Figure. 4 FFO-based parameters identification of DC motor model

\section{DC motor parameters identification}

The proposed FFO-based parameters identification of the DC motor model can be presented by Fig. 4. The root of the mean squared errors (RMSE) between the actual and the calculated speeds are minimized according to the objective function OF as illustrated in Eq. (7). The OF will be fed back to the FFO in order to determine the appropriate DC motor parameters for best fitting the actual motor speed from experimental system with the calculated one based motor model. Previously, Boundaries of motor parameters are identified as introduced in Table 1.

\section{Experimental system}

For the experimental verification of this method, the experimental test bed was built as depicted in Fig. 5. It consists of separately excited DC motor which its rated values are listed in Table 2 . Two variable dc sources are utilized one for exciting the field winding and the other for feeding the armature winding. The control unit 773268901 (a) is used as a test system in the electrical machine laboratory as indicated in Fig. 6. The control unit is a microcontrollercontrolled unit with integrated frequency converter for supplying and controlling the three-phase pendulum machine and displaying the measured values for speed and torque. Digital oscilloscope (HM02024, $200 \mathrm{MHz}, 2 \mathrm{GSa}, 2 \mathrm{MB}$ ) is employed for capture and recording the speed response of the DC motor. Multimeters are utilized for following up the currents and voltages of the armature and field circuits. The speed responses are transmitted from digital oscilloscope through USB to PC for processing. 
Table 1. The boundaries of DC motor parameters

\begin{tabular}{|c|c|c|}
\hline Parameter & Bottom limit & Top limit \\
\hline $\mathrm{R}_{\mathrm{a}}$ & 0.5 & 10 \\
\hline $\mathrm{L}_{\mathrm{a}}$ & 0.01 & 2 \\
\hline $\mathrm{J}$ & 0.001 & 1 \\
\hline $\mathrm{B}$ & 0.0005 & 1 \\
\hline $\mathrm{Kv}$ & 0.01 & 2 \\
\hline
\end{tabular}

Table 2. The electrical specification of DC motor

\begin{tabular}{|c|c|}
\hline Parameter & Rated Value \\
\hline Power & $0.75 \mathrm{Kw}$ \\
\hline Armature Current & $4.8 \mathrm{~A}$ \\
\hline Armature Voltage & $220 \mathrm{~V}$ \\
\hline Field voltage & $200 \mathrm{~V}$ \\
\hline Field current & $0.24 \mathrm{~A}$ \\
\hline Rated Speed & $2000 \mathrm{rpm}$ \\
\hline
\end{tabular}

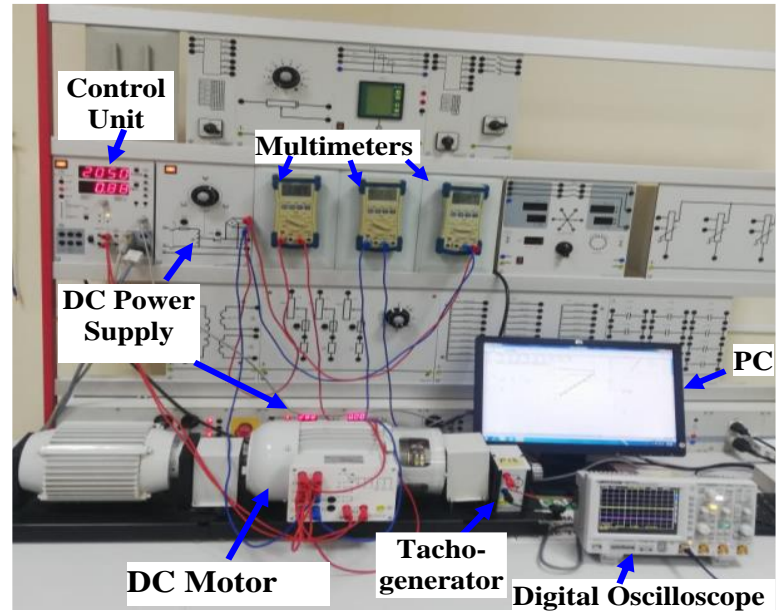

Figure. 5 Experimental system

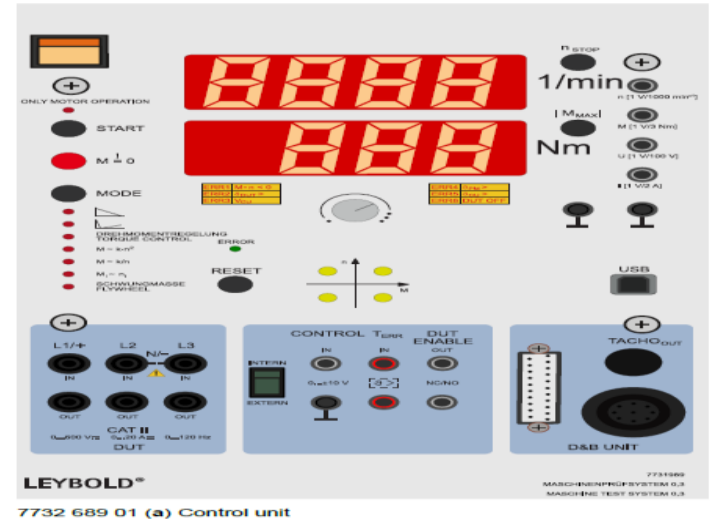

Figure. 6 Control unit

\section{Experimental results and discussion}

To validate the effectiveness of the proposed technique, it is examined at different conditions of the motor operation. Initially, the motor is started with no-load. The steady state motor speed is displayed at the control unit as a digital number in revolution per minute $[\mathrm{rpm}]$. The speed response is measured by the tachogenerator and speed to voltage ratio is calculated $(35 \mathrm{rad} / \mathrm{sec} / \mathrm{V})$. Then, the speed response is captured by the digital oscilloscope as screen shoot (inverted option) as visualized in Fig. 7. Then, the same response is saved as a digital data by the digital oscilloscope (trace-CVS option). The response speed data is formulated by the MATLAB program to appear as shown in Fig. 8.

The FFO algorithm which is coded by MATLAB used this actual speed for comparison with the calculated speed-based model to calculate the OF. After that the OF is returned to the FFO to minimize the error and obtain optimal motor parameters. The parameters of the FFO are set along recommendations of [20], i.e. $n=4, m=25, \alpha=0.4$, and $\beta=0.6$. The speed responses which are calculated by FFO and the actual one are revealed in Fig. 9. The matching between the measured speed and computed speed by FFO approves precision of the gotten DC motor parameters. As soon as the optimizer process stopped, five model parameters of DC motor are efficiently achieved as follow: $R_{a}=3.412 \Omega$, $\mathrm{L}_{\mathrm{a}}=0.0573 \quad \mathrm{H}, \quad \mathrm{J}=0.0424 \quad$ Kg.m2, $\mathrm{B}=0.00267$ N.m.s/rad, $K_{v}=0.1604$ V.s/rad.

In addition, the convergent rate of the RMSE for the proposed technique to identify the parameters is indicated in Fig. 10. It can be observed that the fitness value is kept decreasing until less than 0.0125 after only 200 iterations. After that the FFO results are compared with other competing recent techniquesbased results in the literature. All techniques are chosen for comparison are used the speed response for identification of DC motor parameters as the proposed method. The techniques are employed for comparison are Flower Pollination Algorithm (FPA) [11], Nelder-Mead Simplex (NMS) method [12], Adaptive Tabu Search (ATS) Technique [13] and Wavelet Function (WF) [16]. The fitness value (RMSE) by FFO is found to be the smallest value as shown in Table 3.

To prove the effectiveness of the proposed method, it has been examined at different motor operations such as starting, load change, positive voltage change and negative voltage change as shown in Figs. 11, 12, 13 and 14 respectively. The actual speed responses are captured initially by the digital

Table 3. Comparison between FFO and recent optimization algorithm

\begin{tabular}{|c|c|c|c|c|c|}
\hline Method & FPA & NMS & ATS & WF & FFO \\
\hline $\begin{array}{c}\text { Min- } \\
\text { Fitness } \\
\text { Value }\end{array}$ & 515.9 & 467.5 & 53.6 & 1.07 & 0.0123 \\
\hline $\begin{array}{c}\text { No. } \\
\text { Iterations }\end{array}$ & 500 & 250 & 100 & 100 & 200 \\
\hline
\end{tabular}




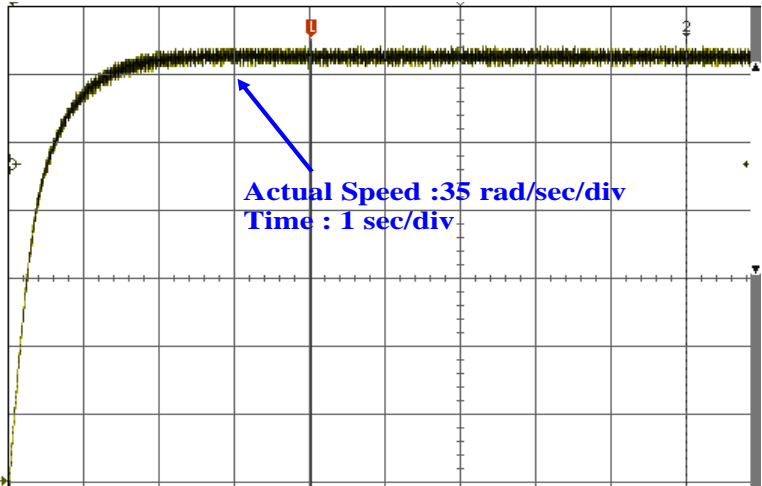

Figure. 7 Actual speed response at starting at $225 \mathrm{rad} / \mathrm{sec}$.

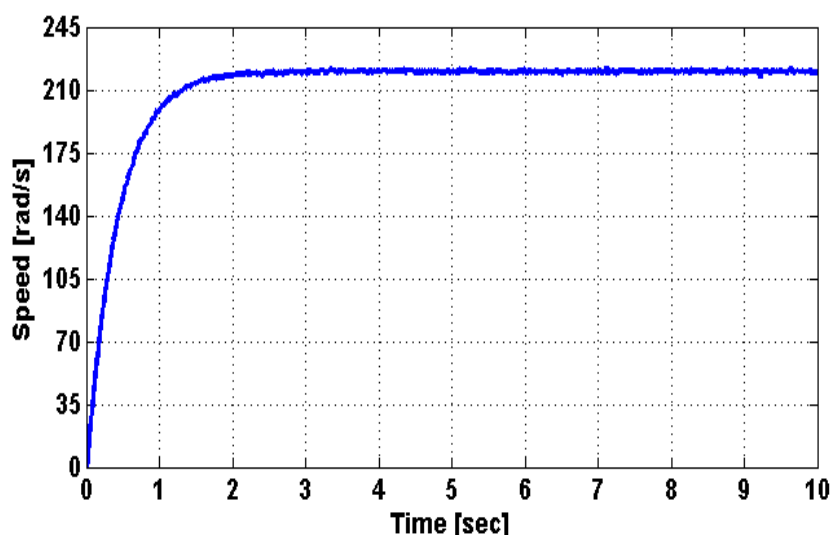

Figure. 8 Actual speed response at starting at $225 \mathrm{rad} / \mathrm{sec}$ after formulated by MATLAB program.

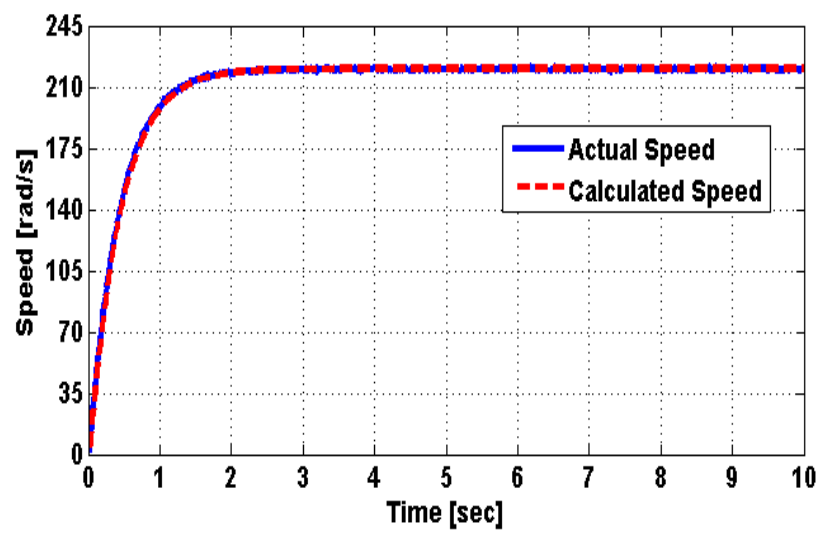

Figure. 9 Comparison between actual speed and FFO calculated speed at starting at $225 \mathrm{rad} / \mathrm{sec}$

oscilloscope and then they are formulated by MATLAB. Then the actual speeds are compared with speed responses based FFO model.

Nearness between the experimental speeds and computed speeds by FFO assures the precision of the deduction DC motor parameters.

\section{Conclusion}

In this research, a novel application of Farmland Fertility Optimizer (FFO) approach is developed for

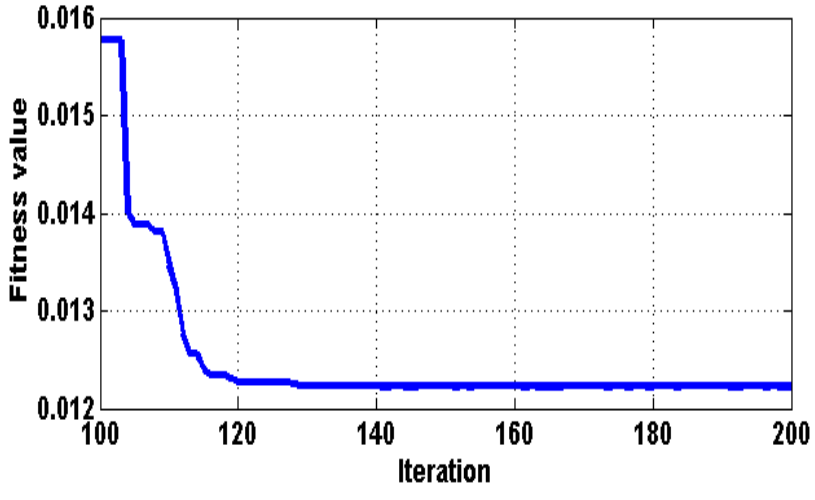

Figure. 10 Convergence rate

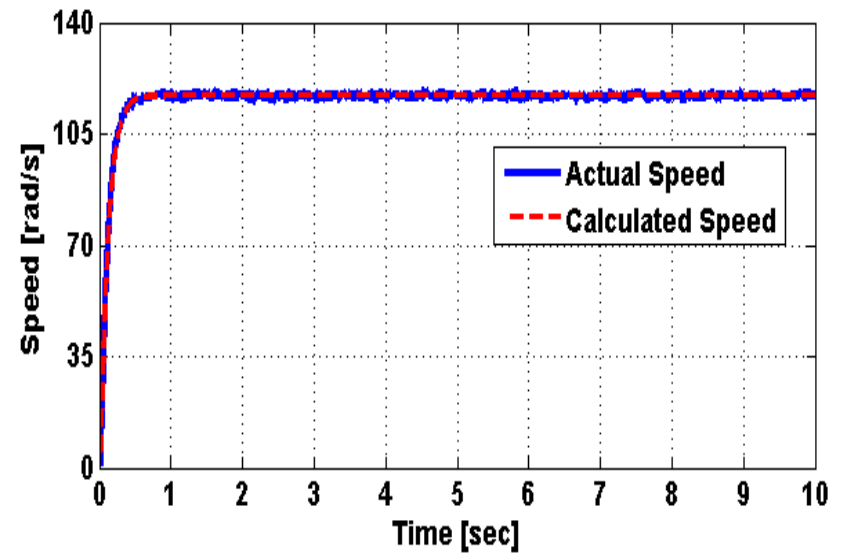

Figure. 11 Comparison between actual speed and FFO calculated speed at starting at $120 \mathrm{rad} / \mathrm{sec}$

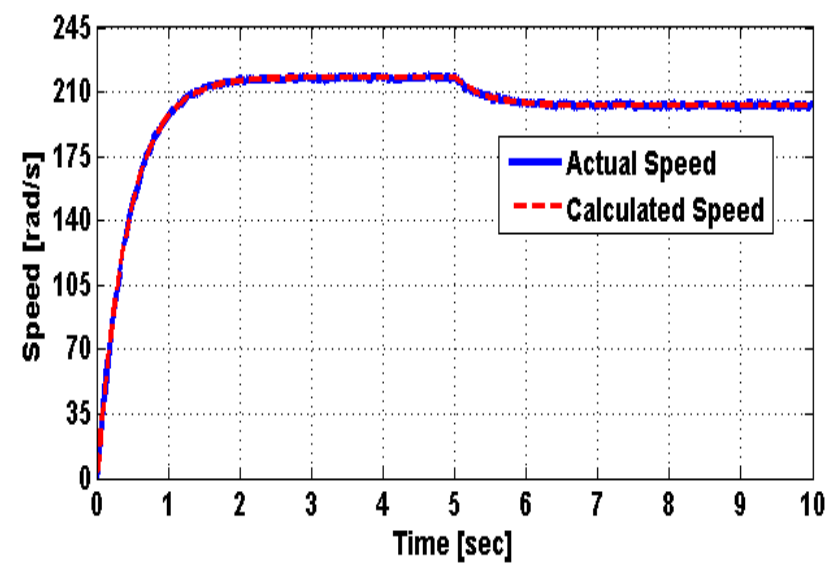

Figure. 12 Comparison between actual speed and FFO calculated speed at load change

identification the parameters of independent excitation DC motor. The methodology, the objective function (OF) and the procedure of FFO are discussed. Minimizing the root mean square errors between the actual speed and calculated speeds is the purpose of the OF for the constraints that are determined by the lower and upper bounds of the parameters. The experimental test bed for the DC motor system is built in the electrical machines laboratory in Northern Border University. A program based MATLAB is 


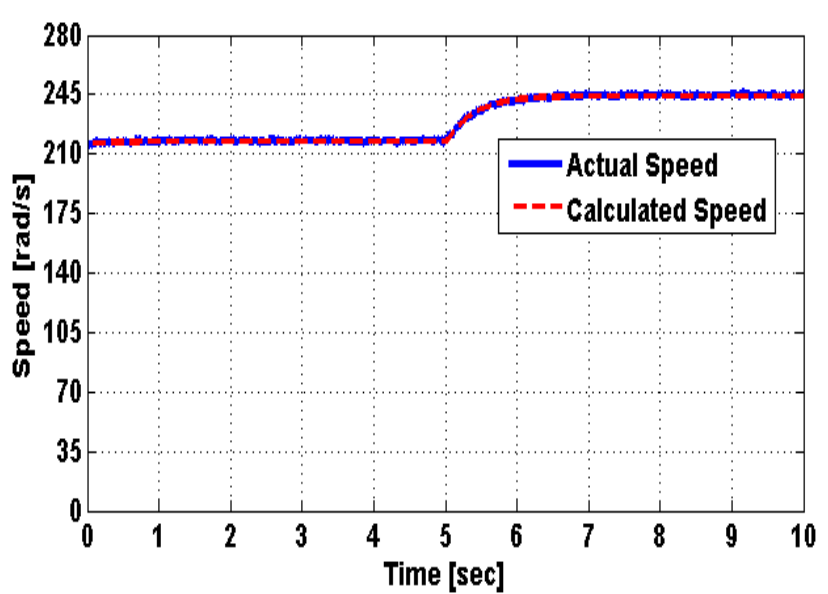

Figure. 13 Comparison between actual speed and FFO calculated speed at positive voltage change

formulated for deducing the actual speed as numerical data. Then, FFO is employed to estimate the parameters of DC motor based the actual speed response. The convergence rate is calculated. A minimum error with fitness value less than 0.0125 after 200 iterations is attained. Furthermore, a comparison between the measured speeds and computed speeds based FFO are carried out at different motor operating conditions. In addition, comparisons between results based FFO and other results based on competing recent methods are introduced. The comparisons show high competition from FFO to other optimization techniques in the literature. Subsequently, the estimated parameters can be used for characterizing the DC motor in dynamic regime and control systems.

\section{Conflicts of Interest}

Author declares that in the preparation of this article there is no conflict of interest.

\section{References}

[1] B. Singh and R. Kumar, "Solar photovoltaic array fed water pump driven by brushless DC motor using Landsman converter", IET Renewable Power Generation, Vol. 10, No. 4, pp. 474- 484, 2016.

[2] H. R. Choi, "Arc Reduction of High-Voltage DC-Motor for Automotive Application by Targetwise Parameter Optimization (TPO)", IEEE Transactions on Magnetics, Vol. 45, No. 3, pp. 1840-1842, 2009.

[3] G. Mirzaeva, R. E. Betz, and T. J. Summers, "Evaluation of Current Density in DC Motor Brushes for Mining Machines Based on Air-Gap Field Measurement", IEEE Transactions on Industry Applications, Vol. 46, No. 4, pp. 12551263, 2010.
[4] C. R. Lee, S. Kim, and C.K. Ahn, "Auto-Tuning Proportional-Type Synchronization Algorithm for DC Motor Speed Control Applications", IEEE Transactions on Circuits and Systems II, Vol. 67, No. 3, pp. 521-525, 2020.

[5] J. Yao, Z. Jiao, and D. Ma," RISE-Based Precision Motion Control of DC Motors with Continuous Friction Compensation", IEEE Transactions on Industrial Electronics, Vol. 61, No. 12, pp. 7067-7075, 2014.

[6] J. Rajagopal and T. Ananthan, "Online System Identification of DC Motor using LabVIEWmyRIO", In: Proc. of International Conf. on Inventive Research in Computing Applications, Coimbatore, India, pp. 528-531, 2018.

[7] M. Li and Y. Ma "Parameter Identification of DC Motor based on Compound Least Square Method", In: Proc. of Fifth Information Technology and Mechatronics Engineering Conf. (ITOEC), Chongqing, China, pp. 11071111, 2020.

[8] A. Rubaai and R. Kotaru, "Online Identification and Control of a DC Motor Using Learning Adaptation of Neural Networks", IEEE Transactions on Industry Applications, Vol. 36, No. 3, pp. 935-942, 2000.

[9] Y. Zhou, Y. C. Soh, and J. X. Shen, "Speed Estimation and Nonmatched Time-varying Parameter Identification for a DC Motor with Hybrid Sliding-mode Observer", IEEE Transactions on Industrial Electronics, Vol. 60, No. 12, pp. 5539- 5549, 2013.

[10] R. Beloiu," Dynamic determination of DC motor parameters - simulation and testing", In: Proc. of International Conference Electronics, Computers and Artificial Intelligence, Bucharest, Romania, pp. 13-17, 2014.

[11] D. Puangdownreong, and S. Hlungnamtip, "Application of Flower Pollination Algorithm to Parameter Identification of DC Motor Model", In: Proc. of Fifth International Electrical Engineering Congress, Pattaya, Thailand, 2017.

[12] M. Dub and R. Jalovecký, "DC Motor Experimental Parameter Identification using the Nelder-Mead Simplex Method", In: Proc. of Fourteen International Power Electronics and Motion Control Conf., EPE-PEMC, pp. 9-11, 2010.

[13] S. Udomsuk, K. L. Areerak, K. N. Areerak and A. Srikaew, "Parameters Identification of Separately Excited DC Motor using Adaptive Tabu Search Technique", In: Proc. of International Conf. on Advances in Energy Engineering, Beijing, China, pp. 48-51, 2010. 
[14] W. Wu, "DC Motor Identification Using Speed Step Responses", In: Proc. of American Control Conf., Waterfront, Baltimore, USA, pp. 19371941, 2010.

[15] N. Patrascoiu, "Identification of parameters for dynamic regime of DC motor through virtual instrumentation", In: Proc. of Fourteen International Conf. on Engineering of Modern Electric Systems (EMES), Oradea, Romania, pp. 192-195, 2017.

[16] R. Parvanova and M. Todorova, "Identification of a DC Motor with Independent Excitation", In: Proc. of International Symposium on Electrical Apparatus \& Technologies (SIELA), Bourgas, Bulgaria, 2020.

[17] M. Lankarany and A. Rezazade, "Parameter Estimation Optimization Based on Genetic Algorithm Applied to DC Motor", In: Proc. of International Conf. on Electrical Engineering, Lahore, Pakistan, 2007.

[18] D. AAZ, E. SI, S. HM, and H. YB, "Modified Farmland Fertility Optimization Algorithm for Optimal Design of a Grid-connected Hybrid Renewable Energy System with Fuel Cell Storage: Case Study of Ataka, Egypt", International Journal of Advanced Computer Science and Applications, Vol. 10, No. 8, pp. 119-132, 2019.

[19] H. Shayanfar and F. Gharehchopogh. "Farmland fertility: A new metaheuristic algorithm for solving continuous optimization problems", Applied Soft Computing, Vol. 71, pp. 728-746, 2018.

[20] A. M. Agwa, A. A. El-Fergany, and H. A. Maksoud, "Electrical Characterization of Photovoltaic Modules using Farmland Fertility Optimizer", Energy Conversion and Management, Vol. 217, 2020. 